\title{
Hippo pathway in intestinal homeostasis and tumorigenesis
}

\author{
Lanfen Chen ${ }^{1 凶}$, Funiu Qin ${ }^{1}$, Xianming Deng ${ }^{1}$, Joseph Avruch ${ }^{2,3}$, Dawang Zhou ${ }^{1 凶}$ \\ ${ }^{1}$ State Key Laboratory of Stress Cell Biology, School of Life Sciences, Xiamen University, Xiamen 361005, China \\ ${ }^{2}$ Department of Molecular Biology, Massachusetts General Hospital, Boston, MA 02114, USA \\ ${ }^{3}$ Department of Medicine, Harvard Medical School, Boston, MA 02115, USA \\ $\triangle$ Correspondence: dwzhou@xmu.edu.cn (D. Zhou), chenlanfen@xmu.edu.cn (L. Chen) \\ Received February 28, 2012 Accepted March 6, 2012
}

\begin{abstract}
The Hippo pathway plays a crucial role in controlling organ size by inhibiting cell proliferation and promoting cell death. Recent findings implicate that this pathway is involved in the process of intestinal regeneration and tumorigenesis. Here we summarize current studies for the function of the Hippo signaling pathway in intestinal homeostasis, regeneration and tumorigenesis, and the crosstalk between the Hippo signaling pathway and other major signaling pathways, i.e. Wnt, Notch and Jak/Stat signaling pathways in intestinal compartment.
\end{abstract}

KEYWORDS Hippo/Mst1/2, YAP/Yorkie, intestinal stem cells

\section{INTRODUCTION}

The primary functions of the intestinal tract are the digestion of food and the absorption of nutrients. Having a direct exposure to the environment, the intestinal tract is frequently challenged by pathogens and other potentially toxic substances contained in food and water. Because of this constant exposure to environmental insults, the intestinal epithelium must be capable of rapid regeneration in the event of tissue damage. Defective regeneration in response to inflammatory and infectious diseases will not only disrupt normal intestinal homeostasis and architecture but is likely to be rapidly fatal (van der Flier and Clevers, 2009). The Hippo pathway, a critical signaling pathway for controlling cell proliferation and apoptosis in response to cell-cell contact, has been shown to play a very important role in both intestinal repair and tumorigenesis (Camargo et al., 2007; Cai et al.,
2010; Karpowicz et al., 2010; Ren et al., 2010; Zhou et al., 2011). The Hippo pathway, originally indentified in Drosophila as a negative regulator of organ size, is highly conserved in mammalian epithelia. As originally defined in Drosophila, the pathway contains a core pair of protein kinases, Hippo (Harvey et al., 2003; Wu et al., 2003) and Warts (Wts)/Lats (Justice et al., 1995), coupled by the scaffold protein Salvador (Sav) (Tapon et al., 2002). The other core elements are the Hippo substrate Mats (Lai et al., 2005), a Warts activator and Yorkie (Yki) (Huang et al., 2005), a Warts/Lats substrate and transcriptional coactivator that binds to the Scalloped/TEAD transcription factor, enhancing expression of proliferative and pro-survival genes. The primary function of the Hippo signaling pathway is to inhibit the activation of Yki, inasmuch as deletion of Yki reverses the overgrowth phenotypes seen with loss of Hippo, Warts/Lats, Salvador or Mats. In mammals, their counterparts are Mst1/2 (Creasy and Chernoff, 1995), Sav1/WW45, Large tumor suppressor (Lats1/2) (St John et al., 1999), Mob1A/B (Luca and Winey, 1998), the Yes-associated protein (Yap) (Sudol, 1994) and its paralogue TAZ, another co-activator (Kanai et al., 2000). Sav1/WW45 is a WW domain-containing scaffold protein that binds Mst1/2 and Lats, through their respective SARAH coiled coil domains, thereby promoting Mst1/2 phosphorylation of Lats (Hwang et al., 2007). Mst1/2 also phosphorylates Mob1A/B (Wei et al., 2007; Praskova et al., 2008), which enhances Mob1's ability to bind and activate Lats1/2. Lats1/2 phosphorylates YAP (or TAZ, depending on the cellular context), which promotes 14-3-3 binding to YAP, causing YAP nuclear exit, and thus inhibition of its function. Loss of Mst1/Mst2 results in a YAP dependent accelerated proliferation, resistance to apoptosis and massive organ overgrowth (Dong et al., 2007; Heallen et al., 2011; Huang et al., 2005; Lee et al., 2010; Lehtinen et al., 2006; Zhou et al., 2008; Zhao et al., 2007, 2008; Lu et al., 
2010; Song et al., 2010; Schlegelmilch et al., 2011), a phenotype that resembles transgenic overexpression of YAP (Ser127Ala) (Camargo et al., 2007). Global expression of this Lats-resistant YAP mutant in mice leads to expansion of progenitor cells in multiple organs (Camargo et al., 2007). Overall, both in flies and mammals, the Hippo pathway plays a crucial role in limiting organ size, by promoting the inhibition of cell proliferation upon cell-cell contact and in suppressing tumorigenesis by inhibiting cell proliferation and promoting cell death

\section{FUNCTION OF THE HIPPO SIGNALING IN INTESTINAL HOMEOSTASIS, REGENERATION AND TUMORIGENESIS}

Partially digested food is released from the stomach into the small intestine for further digestion and nutrient absorption. The functional unit of small intestinal epithelium is the villus, finger-like projections composed of four kinds of differentiated cells that arise from the intestinal stem cells (ISCs) located at the base of the villus, i.e. the crypts of Lieberkühn. In the crypt, ISCs differentiate into transit-amplifying (TA) cells, which thereafter develop into two main types of differentiated cells: the absorptive cells or enterocytes (ECs) and the secretory cells (goblet cells, enteroendocrine (EE) cells, Tuft cells and Paneth cells). The epithelial lining of the intestine is highly dynamic, as those differentiated cells migrate upward from crypt into the villus tip, from which they are shed into the intestinal lumen. The complete turnover time of epithelial cells is about 4 to 5 days. The epithelium of the large intestine also arises from stem cells in crypts but is arranged as a flattened surface without extending villi (Crosnier et al., 2006; Van der Flier et al., 2007; Barker et al., 2010).

In the small intestinal and proximal colonic epithelium of the normal mouse, YAP protein is found in the crypts together with a high abundance of constitutively activated 36-kDa Mst1 polypeptide; the function of YAP is thereby kept largely inactive (Zhou et al., 2011). In the colon, although YAP1 is detectable in the nucleus of crypt cells, elimination of Yap1 does not diminish the abundance of $\mathrm{Ki}^{+} 7^{+}$cells in this compartment, indicating that YAP is not driving the proliferation of these cells (Zhou et al., 2011). Consistent with this view, biallelic deletion of YAP causes no obvious intestinal defects under normal homeostasis (Cai et al., 2010; Zhou et al., 2011). These findings indicate that under normal conditions YAP is largely dispensable and makes little or no contribution to intestinal epithelial proliferation. This situation is different from that in ES and iPS cells, in which YAP is expressed and required for maintaining pluripotency (Lian et al., 2010). However, when the Hippo pathway is inhibited in intestinal epithelia, intestinal epithelial homeostasis is disrupted. Conditional knocking out of Mst2 kinase in the intestinal epithelial cells of Mst1 null mouse (Mst1null/Mst2ff/villin-Cre) results in an expansion of stem-like undifferentiated cells and an al- most complete absence of all secretory lineages (Fig. 1) (Zhou et al., 2011). The loss of Mst1/2 in the intestine decreases phosphorylation of YAP (Ser127 and Ser384) and causes an increase in both YAP abundance and nuclear localization. Mst1null/Mst2ff/villin-Cre mouse phenotype is similar to the transgenic mice overexpressing YAP (Ser127Ala) in the small intestinal compartment, wherein intestinal dysplasia and loss of goblet and Paneth cells are also observed (Camargo et al., 2007). The hyperproliferation and loss of differentiation caused by the MST1/2 deficiency can be entirely reversed by deleting a single YAP allele in Mst1null/ Mst2ff/villin-Cre mouse (Zhou et al., 2011). The deletion of another Hippo pathway component, Salavador/WW45, in mouse intestinal epithelial cells also results in increased YAP abundance and crypt expansion in the affected mice, but to a much milder degree compared to that in the Mst1null/Mst2ff/ villin-Cre mice. The hyperplasia of Savl1-deficient crypts can also be completely reversed by the loss of YAP. However, it is not clear whether Salavador/WW45 deficiency in the intestine has any defects on matured secretory cells (Cai et al., 2010).

The Drosophila midgut, a functional equivalent of the small intestine of mammals, is well characterized, and so provides an attractive model to investigate how intestinal stem cell proliferation and differentiation are regulated (Casali and Batlle, 2009). The simple pseudostratified epithelium of the midgut consists mainly of large polyploidy epithelial cells (ECs), some interspersed diploid EE cells, enteroblasts (EBs), a committed but undifferentiated compartment, and basally located ISC. ISC self-renewal produces an identical daughter ISC along with immature diploid progenitor EB cells, which undergo no further transit divisions and can produce either EC $(90 \%)$ or EE $(10 \%)$ cells. ISC is the only cell in the adult midgut that proliferates (Micchelli and Perrimon, 2006; Ohlstein and Spradling, 2006). As in mouse intestine, the endogenous levels of $\mathrm{Yki}$ in the fly midgut epithelium are not required for maintaining intestinal homeostasis. Warts is normally activated in ECs and needs to be active there to prevent excess ISC proliferation (Staley and Irvine, 2010). Activation of Yki throughout the adult fly resulted in a profound hyperplasia of the intestine with many tightly packed cells and a thicker intestinal epithelium that is accompanied by an apparent increase in ISCs proliferation (Staley and Irvine, 2010). Knockdown of Hippo, Warts, Mer, Expanded (ex) and Fat in ISC produced an increase in ISC/EB frequency and ISC division in the midgut (Karpowicz et al., 2010). However, unlike the mouse intestine, loss of Warts or overexpression of Yki does not block the terminal differentiation, although it does increase the number of proliferating ISCs (Shaw et al., 2010).

Upon exposure to pathogens, chemical and mechanical injuries, or under disease conditions, the damaged intestinal tissue needs to rapidly shift from normal epithelial turnover to regeneration. This transition has to be well controlled or it 
A

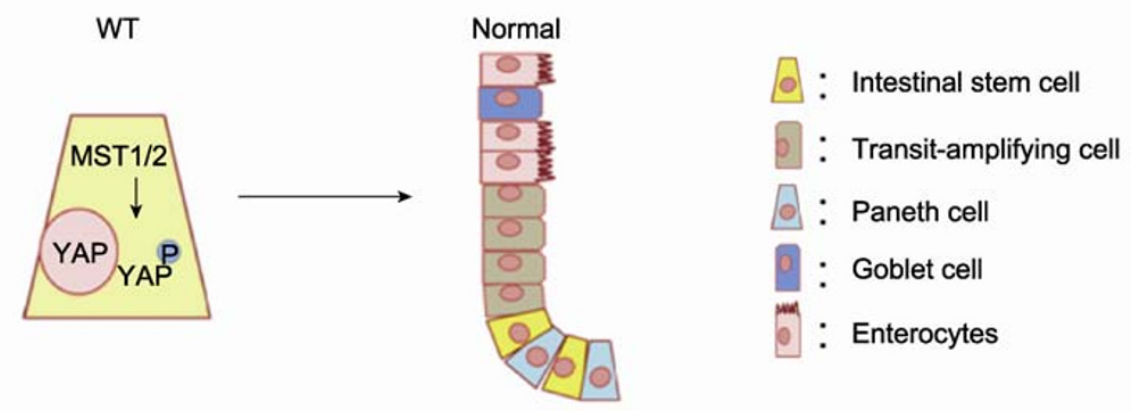

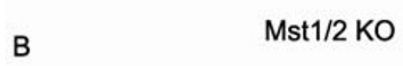

Expansion of stem-like
undifferentiated cells

B

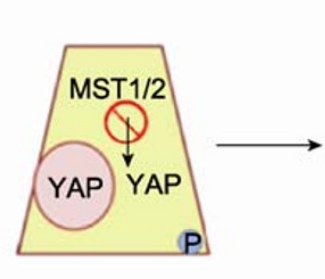

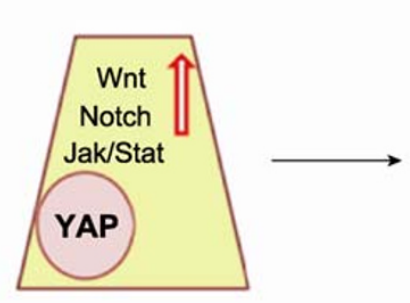

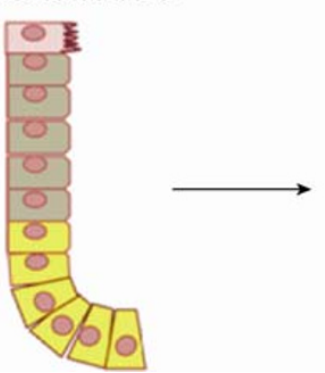

Adenomas

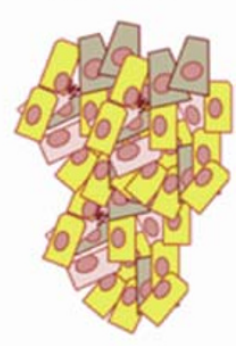

Figure 1. Hippo signaling pathway controls intestinal homeostasis and tumorigenesis. (A) In the normal developed intestine, the constitutive activation of the Hippo pathway maintains inactivation of YAP, which is dispensable for the intestinal epithelial proliferation under normal condition. (B) In the Mst1/2 null intestine, inactivation of the Hippo signaling pathway increases the activity and abundance of YAP in nucleus, and consequently enhances the Wnt, Notch, and Jak/Stat signaling pathways, which results in an expansion of stem-like undifferentiated cells and almost complete absence of all secretary lineages, and eventual development of colonic adenomas.

could result in pathological conditions; if insufficient, it results in tissue atrophy and deterioration, whereas excessive or unrestrained regeneration may foster tumor growth. Inhibition of the Hippo signaling pathway appears to be a central feature of the intestinal regenerative response. Although YAP is dispensable for the normal mouse intestinal homeostasis, it is required for tissue regeneration caused by injury (Cai et al., 2010). YAP protein is dramatically increased in the crypts of the recovery phase from the DSS treated mice. Following the DSS treatment, the conditional knock out of YAP with villin-Cre mouse shows a dramatic increase in mortality rate and rapid decrease in body weight compared with the control littermate (Ren et al., 2010). Overexpression or activation of Yki in ISCs or ECs can both increase ISC proliferation. Yki is specifically required in ISCS/EBs for the ISC proliferation stimulated by DSS in flies (Ren et al., 2010), and it can also be activated in ECs by the DNA damaging agent bleomycin, which further induces a regenerative response in the ISC by a nonautonomous manner (Staley and Irvine, 2010).

Colon cancer is commonly associated with persistent inflammatory states and the consequent ongoing regenerative response. Loss of control over normal tissue repair or renewal mechanisms will lead to malignant transformation (van der Flier and Clevers, 2009; Terzic et al., 2010). YAP is fre- quently overexpressed in common human cancers (Steinhardt et al., 2008; Zhou et al., 2009; Wang et al., 2011; Zhou et al., 2011). The activation of YAP/Yki for regulating ISC regeneration in response to environmental challenge indicates that a deficiency of Hippo signaling may also contribute to tumorigenesis in intestines. Indeed, the mice with Mst1/Mst2 deletion in intestinal epithelia develop colonic adenomas within 3 months of birth; this rapid occurrence suggests that those polyps might evolve into invasive and/or metastatic cancer, an outcome precluded by the wasting and early mortality of these mice (Fig. 1) (Zhou et al., 2011). Mice lacking intestinal expression of Sal/WW45 show a milder phenotype with an average of 1-2 adenomas at 13 months. However, when exposed to DSS-induced injury and repair, the mice with Sav1-deficient intestinal epithelium show a greatly exacerbated tumorigenesis compared with DSStreated normal mice, and this enhanced tumorigenicity is YAP dependent (Cai et al., 2010). Both studies show that adenoma development in the colon depends on the activation of YAP. Notably, a very high prevalence of Yap1 overexpression is found in human colonic cancers and derived cancer cell lines (Steinhardt et al., 2008; Zhou et al., 2011). Depletion of Yap1 in colon cancer-derived cell lines that overexpress Yap1 results in a strong inhibition of their proliferation in vitro 
and suggests that Yap1 is likely to be an important driver in human colon cancers (Zhou et al., 2011).

\section{EFFECTS OF THE HIPPO PATHWAY ON WNT, NOTCH AND JAK/STAT SIGNALING PATHWAYS IN INTESTINAL COMPARTMENTS}

Several conserved signaling pathways including Notch, Wnt and Jak/Stat regulate the maintenance, proliferation, and differentiation of ISCs (Jarriault et al., 1995; Lin et al., 2008; Jiang et al., 2009; Miyamoto and Rosenberg, 2011). Classical Wnt signaling and $\beta$-catenin transcritional activity are very important for the maintenance and expansion of undifferentiated progenitor cells in the small intestine. Notch signaling assists Wnt in promoting ISC proliferation and also independently negatively regulates epithelial differentiation and maintenances of undifferentiated progenitor/stem cells. In contrast, the Jak/Stat signaling pathway is the critical mediator of damage-induced ISC proliferation.

In Drosophila, Yki has been shown to bind and presumably sequester Dishevelled (Dsh) in the cytoplasm, thereby interfering with the ability of Wnt to disrupt the $\beta$-catenin destruction complex, and then further promote activation of Armadillo, the fly $\beta$-catenin orthologue (Varelas et al., 2010). The inactivation of the Hippo pathway to promote the hyperproliferation of intestinal stem cells and to inhibit intestinal epithelial differentiation is attributed largely to an enhancement of $\beta$-catenin action and activation of Notch signaling. In Mst1null/Mst2ff/villin-Cre mice, although the overall abundance of nuclear $\beta$-catenin in the intestine is unchanged, the $\beta$-catenin transcriptional efficacy is increased which is evident by the increased abundance of the activated form of $\beta$-catenin (dephosphoSer37/Thr41) and Wnt targets Lgr5 and Ascl2, both of which are stem cell markers in the intestine (Zhou et al., 2011). Camargo et al. also showed that YAP1induced intestinal dysplasia is accompanied with increased amounts of nuclear $\beta$-catenin (Camargo et al., 2007). The overexpression of activated YAP1 in the intestine results in an expansion of the compartment expressing the EphB2 receptor, which is the target gene of the Wnt pathway and is involved in correctly positioning Paneth cells (Camargo et al., 2007). Depletion of YAP in SW480 cells reduces $\beta$-catenin dependent transcriptional activity by over $80 \%$ (Zhou et al., 2011). In Caco colorectal cancer (CRC) cell lines, endogenous YAP can be co-precipitated with $\beta$-catenin and phosphorylation of YAP at Ser127 is required for this association. $\beta$-catenin is retained in the cytoplasm through its binding to the phosphorylated YAP. Furthermore, compared to CRCs with high activity of Hippo signaling or normal colon cells, CRCs with low activity of Hippo signaling have increased expression levels of $\beta$-catenin/TCF-target genes (ENC1, MYC, SOX9, CD44, LGR5, and ASCL2), which play a crucial role in controlling intestinal homeostasis (Imajo et al., 2012). Thus, the Hippo pathway might inhibit multiple steps in Wnt/ $\beta$-catenin signaling: (1) Dvl phosphorylation, (2) nuclear accumulation of $\beta$-catenin, and (3) transcription of $\beta$-catenin/ TCF-target genes (Varelas et al., 2010; Imajo et al., 2012).

Independently of Wnt signaling, Notch plays an essential role in regulating epithelial differentiation and maintaining stem and progenitor cells in the intestine (Jarriault et al., 1995; Miyamoto and Rosenberg, 2011). The absence of differentiated cells in the Mst1/2 null intestine or the Yap overexpressed intestine suggests activation of the Notch signaling pathway results from the inactivation of Hippo signaling (Zhou et al., 2011). Hairy and enhancer of split 1 (Hes1), a Notch target gene, is normally expressed only by the undifferentiated crypt progenitor cell population; however, the Hes1 expressing cell compartment was expanded to all epithelial cells along the villi in mice transgenically overexpressing YAP (ser127Ala) (Camargo et al., 2007). In these transgenic mice, induction of YAP1 in the presence of the $\mathrm{Y}$-secretase inhibitor, dipenzazepine (DBZ), leads to a much less dysplastic phenotype in the intestine with decreased proliferation and the presence of goblet cells and differentiated enterocytes (Camargo et al., 2007). Thus, YAP1-mediated expansion of intestinal progenitor cells at least partially relies on activation of the Notch signaling pathway. Strong activation of the Notch signaling pathway is also evident in the intestine of Mst1null/ Mst2ff/villin-Cre mouse. The increased expression of the Notch ligand Jagged 1, mediated possibly in part through up-regulated Wnt signaling (Pannequin et al., 2009; Rodilla et al., 2009), is found in Mst1/Mst2 deficient intestine. The intranuclear Notch intracellular domain (NICD) is increased along the entire epithelial surface of the Mst1/2 null intestine, and furthermore, the abundance of Hes 1 is also up-regulated in both the small and large intestines of this mouse (Zhou et al., 2011).

In Drosophila, when ECs are subjected to apoptosis, enteric infection, or JNK-mediated stress signaling, they produce cytokines (Upd, Upd2, and Upd3) that activate Jak/Stat signaling in ISCs, promoting their rapid division (Jiang et al., 2009). The increased injury-induced JNK signaling can also drive Yki activation. Warts RNAi or Yki overexpresion in ECs induced an increase in the expression of Socs36E, a Jak/Stat pathway target gene, suggesting that loss of Hippo signaling or excessive Yki activity might result in elevated Jak/Stat (Ren et al., 2010). These findings suggest a link between these two pathways in mediating the proliferative stress response. Hippo signaling exhibits both autonomous and nonautonomous influence on the proliferation of ISCs, although either the autonomous or non-autonomous effect is more emphasized in certain studies using different experimental systems (Karpowicz et al., 2010; Ren et al., 2010; Shaw et al., 2010; Staley and Irvine, 2010). Activation of Yki in ECs promotes ISC proliferation and intestinal hyperplasia by inducing expression of Upd genes which are secreted from ECs and then activate the Jak/Stat pathway in ISCs to promote their proliferation and differentiation. On the other hand, when Yki 
is overexpressed in ISCs, it also regulates the expression of the upd cytokines in ISCs, and then activates Jak/Stat signaling in ISCs themselves. Loss of Jak/Stat signaling in ISC can rescue the Yki overexpression phenotype (Karpowicz et al., 2010; Shaw et al., 2010). The same as Yap in the mouse (Cai et al., 2010), Yki is also specifically required in ISCs/EBs for DSS to stimulate ISC proliferation, although Ren et al. found that loss of Hippo signaling in ECS but not in ISC can cooperate with DSS to stimulate ISC proliferation (Ren et al., 2010).

\section{CONCLUSION}

The Hippo tumor suppresser pathway is widely involved in intestinal development, regeneration and tumorigenesis. A full understanding of the Hippo pathway and its interaction with other critical signaling pathways, i.e. Notch, Wnt or Jak/Stat pathways, for maintaining normal intestinal homeostasis and function will shed a new light on therapeutic strategies for the treatment of intestinal diseases.

\section{ACKNOWLEDGEMENTS}

DZ was supported by grants from the China's 1000 Young Talents Program, the 111 Project of The Ministration of Education of China (No. B06016), the Fundamental Research Funds for the Central Universities of China (No. 2010111079), National Natural Science Foundation of China (Grant No. 81101503) and Natural Science Foundation of Fujian (No. 2011J05096). JA was in part supported by the RO1 awards CA136567 (JA), DK17776 (JA) and institutional funds. The authors have no financial conflicts to disclose.

\section{REFERENCES}

Barker, N., Bartfeld, S., and Clevers, H. (2010). Tissue-resident adult stem cell populations of rapidly self-renewing organs. Cell Stem Cell 7, 656-670.

Cai, J., Zhang, N., Zheng, Y., de Wilde, R.F., Maitra, A., and Pan, D. (2010). The Hippo signaling pathway restricts the oncogenic potential of an intestinal regeneration program. Genes Dev 24, 2383-2388.

Camargo, F.D., Gokhale, S., Johnnidis, J.B., Fu, D., Bell, G.W., Jaenisch, R., and Brummelkamp, T.R. (2007). YAP1 increases organ size and expands undifferentiated progenitor cells. Curr Biol 17, 2054-2060.

Casali, A., and Batlle, E. (2009). Intestinal stem cells in mammals and Drosophila. Cell Stem Cell 4, 124-127.

Creasy, C.L., and Chernoff, J. (1995). Cloning and characterization of a member of the MST subfamily of Ste20-like kinases. Gene 167, 303-306.

Crosnier, C., Stamataki, D., and Lewis, J. (2006). Organizing cell renewal in the intestine: stem cells, signals and combinatorial control. Nat Rev Genet 7, 349-359.

Dong, J., Feldmann, G., Huang, J., Wu, S., Zhang, N., Comerford, S.A., Gayyed, M.F., Anders, R.A., Maitra, A., and Pan, D. (2007).
Elucidation of a universal size-control mechanism in Drosophila and mammals. Cell 130, 1120-1133.

Harvey, K.F., Pfleger, C.M., and Hariharan, I.K. (2003). The Drosophila Mst ortholog, hippo, restricts growth and cell proliferation and promotes apoptosis. Cell 114, 457-467.

Heallen, T., Zhang, M., Wang, J., Bonilla-Claudio, M., Klysik, E., Johnson, R.L., and Martin, J.F. (2011). Hippo pathway inhibits Wnt signaling to restrain cardiomyocyte proliferation and heart size. Science 332, 458-461.

Huang, J., Wu, S., Barrera, J., Matthews, K., and Pan, D. (2005). The Hippo signaling pathway coordinately regulates cell proliferation and apoptosis by inactivating Yorkie, the Drosophila Homolog of YAP. Cell 122, 421-434.

Hwang, E., Ryu, K.S., Pääkkönen, K., Güntert, P., Cheong, H.K., Lim, D.S., Lee, J.O., Jeon, Y.H., and Cheong, C. (2007). Structural insight into dimeric interaction of the SARAH domains from Mst1 and RASSF family proteins in the apoptosis pathway. Proc Natl Acad Sci U S A 104, 9236-9241.

Imajo, M., Miyatake, K., limura, A., Miyamoto, A., and Nishida, E. (2012). A molecular mechanism that links Hippo signalling to the inhibition of Wnt/beta-catenin signalling. EMBO J 31, 1109-1122.

Jarriault, S., Brou, C., Logeat, F., Schroeter, E.H., Kopan, R., and Israel, A. (1995). Signalling downstream of activated mammalian Notch. Nature 377, 355-358.

Jiang, H., Patel, P.H., Kohlmaier, A., Grenley, M.O., McEwen, D.G., and Edgar, B.A. (2009). Cytokine/Jak/Stat signaling mediates regeneration and homeostasis in the Drosophila midgut. Cell 137, 1343-1355.

Justice, R.W., Zilian, O., Woods, D.F., Noll, M., and Bryant, P.J. (1995). The Drosophila tumor suppressor gene warts encodes a homolog of human myotonic dystrophy kinase and is required for the control of cell shape and proliferation. Genes Dev 9, 534-546.

Kanai, F., Marignani, P.A., Sarbassova, D., Yagi, R., Hall, R.A., Donowitz, M., Hisaminato, A., Fujiwara, T., Ito, Y., Cantley, L.C., et al. (2000). TAZ: a novel transcriptional co-activator regulated by interactions with 14-3-3 and PDZ domain proteins. EMBO J 19, 6778-6791.

Karpowicz, P., Perez, J., and Perrimon, N. (2010). The Hippo tumor suppressor pathway regulates intestinal stem cell regeneration. Development 137, 4135-4145.

Lai, Z.C., Wei, X., Shimizu, T., Ramos, E., Rohrbaugh, M., Nikolaidis, N., Ho, L.L., and Li, Y. (2005). Control of cell proliferation and apoptosis by mob as tumor suppressor, mats. Cell 120, 675-685.

Lee, K.P., Lee, J.H., Kim, T.S., Kim, T.H., Park, H.D., Byun, J.S., Kim, M.C., Jeong, W.I., Calvisi, D.F., Kim, J.M., et al. (2010). The Hippo-Salvador pathway restrains hepatic oval cell proliferation, liver size, and liver tumorigenesis. Proc Natl Acad Sci U S A 107, 8248-8253.

Lehtinen, M.K., Yuan, Z., Boag, P.R., Yang, Y., Villén, J., Becker, E.B., DiBacco, S., de la Iglesia, N., Gygi, S., Blackwell, T.K., et al. (2006). A conserved MST-FOXO signaling pathway mediates oxidative-stress responses and extends life span. Cell 125, 987-1001.

Lian, I., Kim, J., Okazawa, H., Zhao, J., Zhao, B., Yu, J., Chinnaiyan, A., Israel, M.A., Goldstein, L.S., Abujarour, R., et al. (2010). The role of YAP transcription coactivator in regulating stem cell self-renewal and differentiation. Genes Dev 24, 1106-1118. 
Lin, G., Xu, N., and Xi, R. (2008). Paracrine Wingless signalling controls self-renewal of Drosophila intestinal stem cells. Nature 455, 1119-1123.

Lu, L., Li, Y., Kim, S.M., Bossuyt, W., Liu, P., Qiu, Q., Wang, Y., Halder, G., Finegold, M.J., Lee, J.S., et al. (2010). Hippo signaling is a potent in vivo growth and tumor suppressor pathway in the mammalian liver. Proc Natl Acad Sci U S A 107, 1437-1442.

Luca, F.C., and Winey, M. (1998). MOB1, an essential yeast gene required for completion of mitosis and maintenance of ploidy. Mol Biol Cell 9, 29-46.

Micchelli, C.A., and Perrimon, N. (2006). Evidence that stem cells reside in the adult Drosophila midgut epithelium. Nature 439, 475-479.

Miyamoto, S., and Rosenberg, D.W. (2011). Role of Notch signaling in colon homeostasis and carcinogenesis. Cancer Sci 102, 1938-1942.

Ohlstein, B., and Spradling, A. (2006). The adult Drosophila posterior midgut is maintained by pluripotent stem cells. Nature 439, 470-474.

Pannequin, J., Bonnans, C., Delaunay, N., Ryan, J., Bourgaux, J.F., Joubert, D., and Hollande, F. (2009). The wnt target jagged-1 mediates the activation of notch signaling by progastrin in human colorectal cancer cells. Cancer Res 69, 6065-6073.

Praskova, M., Xia, F., and Avruch, J. (2008). MOBKL1A/MOBKL1B phosphorylation by MST1 and MST2 inhibits cell proliferation. Curr Biol 18, 311-321.

Ren, F., Wang, B., Yue, T., Yun, E.Y., Ip, Y.T., and Jiang, J. (2010). Hippo signaling regulates Drosophila intestine stem cell proliferation through multiple pathways. Proc Natl Acad Sci U S A 107, 21064-21069.

Rodilla, V., Villanueva, A., Obrador-Hevia, A., Robert-Moreno, A., Fernández-Majada, V., Grilli, A., López-Bigas, N., Bellora, N., Albà, M.M., Torres, F., et al. (2009). Jagged1 is the pathological link between Wnt and Notch pathways in colorectal cancer. Proc Natl Acad Sci U S A 106, 6315-6320.

Schlegelmilch, K., Mohseni, M., Kirak, O., Pruszak, J., Rodriguez, J.R., Zhou, D., Kreger, B.T., Vasioukhin, V., Avruch, J., Brummelkamp, T.R., et al. (2011). Yap1 acts downstream of -catenin to control epidermal proliferation. Cell 144, 782-795.

Shaw, R.L., Kohlmaier, A., Polesello, C., Veelken, C., Edgar, B.A., and Tapon, N. (2010). The Hippo pathway regulates intestinal stem cell proliferation during Drosophila adult midgut regeneration. Development 137, 4147-4158.

Song, H., Mak, K.K., Topol, L., Yun, K., Hu, J., Garrett, L., Chen, Y., Park, O., Chang, J., Simpson, R.M., et al. (2010). Mammalian Mst1 and Mst2 kinases play essential roles in organ size control and tumor suppression. Proc Natl Acad Sci U S A 107, 1431-1436.

St John, M.A., Tao, W., Fei, X., Fukumoto, R., Carcangiu, M.L., Brownstein, D.G., Parlow, A.F., McGrath, J., and Xu, T. (1999). Mice deficient of Lats1 develop soft-tissue sarcomas, ovarian tumours and pituitary dysfunction. Nat Genet 21, 182-186.

Staley, B.K., and Irvine, K.D. (2010). Warts and Yorkie mediate intestinal regeneration by influencing stem cell proliferation. Curr Biol 20, 1580-1587.

Steinhardt, A.A., Gayyed, M.F., Klein, A.P., Dong, J., Maitra, A., Pan, D., Montgomery, E.A., and Anders, R.A. (2008). Expression of
Yes-associated protein in common solid tumors. Hum Pathol 39, 1582-1589.

Sudol, M. (1994). Yes-associated protein (YAP65) is a proline-rich phosphoprotein that binds to the $\mathrm{SH} 3$ domain of the Yes proto-oncogene product. Oncogene 9, 2145-2152.

Tapon, N., Harvey, K.F., Bell, D.W., Wahrer, D.C., Schiripo, T.A., Haber, D.A., and Hariharan, I.K. (2002). salvador Promotes both cell cycle exit and apoptosis in Drosophila and is mutated in human cancer cell lines. Cell 110, 467-478.

Terzic, J., Grivennikov, S., Karin, E., and Karin, M. (2010). Inflammation and colon cancer. Gastroenterology 138, 2101-2114 e2105.

van der Flier, L.G., and Clevers, H. (2009). Stem cells, self-renewal, and differentiation in the intestinal epithelium. Annu Rev Physiol 71, 241-260.

Van der Flier, L.G., Sabates-Bellver, J., Oving, I., Haegebarth, A., De Palo, M., Anti, M., Van Gijn, M.E., Suijkerbuijk, S., Van de Wetering, M., Marra, G., et al. (2007). The Intestinal Wnt/TCF Signature. Gastroenterology 132, 628-632.

Varelas, X., Miller, B.W., Sopko, R., Song, S., Gregorieff, A., Fellouse, F.A., Sakuma, R., Pawson, T., Hunziker, W., McNeill, H., et al. (2010). The Hippo pathway regulates Wnt/beta-catenin signaling. Dev Cell 18, 579-591.

Wang, X., Su, L., and Ou, Q. (2011). Yes-associated protein promotes tumour development in luminal epithelial derived breast cancer. Eur J Cancer. 2011 Nov 4. [Epub ahead of print]

Wei, X., Shimizu, T., and Lai, Z.C. (2007). Mob as tumor suppressor is activated by Hippo kinase for growth inhibition in Drosophila. EMBO J 26, 1772-1781.

Wu, S., Huang, J., Dong, J., and Pan, D. (2003). hippo encodes a Ste-20 family protein kinase that restricts cell proliferation and promotes apoptosis in conjunction with salvador and warts. Cell 114, 445-456.

Zhao, B., Wei, X., Li, W., Udan, R. S., Yang, Q., Kim, J., Ikenoue, T., Yu, J., Li, L., et al. (2007). Inactivation of YAP oncoprotein by the Hippo pathway is involved in cell contact inhibition and tissue growth control. Genes Dev 21, 2747-2761.

Zhao, B., Ye, X., Yu, J., Li, L., Li, W., Li, S., Yu, J., Lin, J. D., Wang, C. Y., Chinnaiyan, A. M., et al. (2008). TEAD mediates YAP-dependent gene induction and growth control. Genes Dev 22, 1962-1971.

Zhou, D., Conrad, C., Xia, F., Park, J.S., Payer, B., Yin, Y., Lauwers, G.Y., Thasler, W., Lee, J.T., Avruch, J., et al. (2009). Mst1 and Mst2 maintain hepatocyte quiescence and suppress hepatocellular carcinoma development through inactivation of the Yap1 oncogene. Cancer Cell 16, 425-438.

Zhou, D., Medoff, B.D., Chen, L., Li, L., Zhang, X.F., Praskova, M., Liu, M., Landry, A., Blumberg, R.S., Boussiotis, V.A., et al. (2008). The Nore1B/Mst1 complex restrains antigen receptor-induced proliferation of naïve T cells. Proc Natl Acad Sci U S A 105, 20321-20326.

Zhou, D., Zhang, Y., Wu, H., Barry, E., Yin, Y., Lawrence, E., Dawson, D., Willis, J.E., Markowitz, S.D., Camargo, F.D., et al. (2011). Mst1 and Mst2 protein kinases restrain intestinal stem cell proliferation and colonic tumorigenesis by inhibition of Yes-associated protein (Yap) overabundance. Proc Natl Acad Sci U S A 108, E1312-E1320. 